\title{
Characterization of bacterial-type phosphoeno/pyruvate carboxylase expressed in male gametophyte of higher plants
}

\author{
Tomoko Igawa ${ }^{1,4}$, Masayuki Fujiwara'1, Ichiro Tanaka², Yoichiro Fukao', Yuki Yanagawa ${ }^{1,3^{*}}$
}

\begin{abstract}
Background: Phosphoeno/pyruvate carboxylase (PEPC) is a critical enzyme catalyzing the $\beta$-carboxylation of phosphoeno/pyruvate (PEP) to oxaloacetate, a tricarboxylic acid (TCA) cycle intermediate. PEPC typically exists as a Class-1 PEPC homotetramer composed of plant-type PEPC (PTPC) polypeptides, and two of the subunits were reported to be monoubiquitinated in germinating castor oil seeds. By the large-scale purification of ubiquitin (Ub)related proteins from lily anther, two types of PEPCs, bacterial-type PEPC (BTPC) and plant-type PEPC (PTPC), were identified in our study as candidate Ub-related proteins. Until now, there has been no information about the properties of the PEPCs expressed in male reproductive tissues of higher plants.

Results: Expression analyses showed that lily BTPC (LIBTPC) and Arabidopsis BTPC (AtBTPC) were significantly expressed in pollen. The fusion protein AtBTPC-Venus localized in the cytoplasm of the vegetative cell (VC). Both LIBTPC and AtBTPC expression initiated after the last mitosis before pollen germination. Lily PTPC (LIPTPC) and monoubiquitinated LIPTPC (Ub-LIPTPC) remained at constant levels during pollen development. In late bicellular pollen of lily, LIBTPC forms a hetero-octameric Class-2 PEPC complex with LIPTPC to express PEPC activity.

Conclusion: Our results suggest that an LIBTPC:Ub-LIPTPC:LIPTPC complex is formed in the VC cytoplasm during late pollen development. Both LIBTPC and AtBTPC expression patterns are similar to the patterns of the appearance of storage organelles during pollen development in lily and Arabidopsis, respectively. Therefore, BTPC is thought to accelerate the metabolic flow for the synthesis of storage substances during pollen maturation. Our study provides the first characterization of BTPC in pollen, the male gametophyte of higher plants.
\end{abstract}

\section{Background}

Phosphoenolpyruvate carboxylase (PEPC, EC4.1.1.31) catalyzes the irreversible $\beta$-carboxylation of phosphoenolpyruvate (PEP) to yield oxaloacetate and inorganic phosphate (Additional file 1). PEPC exists widely in plants, algae, and bacteria, but not in animals or fungi [1]. In plants, PEPC acts as an allosteric enzyme and is phosphorylated by PEPC protein kinase [1-3]. Active PEPC commonly consists of a plant-type PEPC (PTPC) homotetramer, and is typically inhibited by L-malate and aspartic acid and activated by glucose-6-phosphate (Glc-6-P). PEPC has been extensively studied in C4 and

\footnotetext{
* Correspondence: yyana@affrc.go.jp

${ }^{1}$ The Plant Science Education Unit, The Graduate School of Biological Sciences, Nara Institute of Science and Technology, 8916-5 Takayama-cho, Ikoma, Nara 630-0101, Japan

Full list of author information is available at the end of the article
}

CAM photosynthesis, because it is a critical enzyme catalyzing the initial reaction of atmospheric $\mathrm{CO}_{2}$ fixation [1]. It also plays pivotal metabolic roles in nonphotosynthetic and $\mathrm{C} 3$ photosynthetic cells, particularly in the anaplerotic replenishment of the TCA cycle intermediates consumed during lipid synthesis [4], biosynthesis, and nitrogen assimilation [5]. The genomic analysis of the PEPC of Arabidopsis and rice first revealed that higher plants contain a small PEPC family containing two types of PEPC, PTPC and bacterial-type PEPC (BTPC) [6]. BTPC resembles the bacterial PEPC rather than the common plant PEPC in terms of its gene structure and the absence of an N-terminal seryl-phosphorylation domain, a hallmark of PTPC (Additional file 2). Recent studies have indicated that BTPC in developing castor oil seeds (COS) interacts with PTPC to form a heterooctameric complex with PEPC activity $[7,8]$. 
Recently, PTPC was reported to be a ubiquitinated protein in Arabidopsis [9,10] and a monoubiquitinated protein in germinated COS [11]. Ubiquitination is one of the major protein modifications that occur in all eukaryotic cells, and is critical for the regulation of various cellular functions, such as DNA damage repair, endocytosis, endosomal sorting, and signal transduction, in addition to proteolysis by the $26 \mathrm{~S}$ proteasome [12]. We previously established a method for the purification and identification of Ub-related proteins (ubiquitinated proteins and their associated proteins) in Arabidopsis seedlings [13]. Applying this method, we purified and identified PTPC and BTPC from the lily anther as candidate Ub-related proteins (Additional files 3 and 4).

As far as we know, no previous study has focused on the PEPC expressed in plant reproductive tissues. Because AtBTPC showed significant expression in stamen (Figure 1) and COS BTPC is reported to interact with PTPC $[7,8]$, we focused here on BTPC and PTPC in male reproductive tissues. In this study, we analyzed the expression, localization, and interaction of BTPC and PTPC in lily and Arabidopsis. Our results suggest that BTPC forms a complex with PTPC and monoubiquitinated PTPC (Ub-PTPC) to accelerate the accumulation of storage substances during pollen maturation.

\section{Results}

BTPC and PTPC are identified as Ub-related proteins in lily anther, and AtBTPC shows stamen-specific expression With the large-scale purification of Ub-related proteins from the lily anther, 13 proteins were identified as candidate Ub-related proteins by liquid chromatographytandem mass spectrometry (LC-MS/MS). The limited number of proteins identified is probably attributable to

\begin{tabular}{|c|c|c|c|c|c|c|c|c|}
\hline & 12 & 4 & 5 & 6 & 7 & 8 & 9 & 10 \\
\hline $\begin{array}{l}\text { At1g78900 (VHA-A) } \\
\text { Gene ID: } 844228\end{array}$ & & 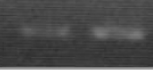 & $=$ & $\omega$ & 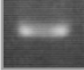 & 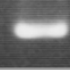 & - & 0 \\
\hline $\begin{array}{l}\text { At2g07698 (T18C6.2) } \\
\text { Gene ID: } 815374\end{array}$ & & & & bysis & 8 & - & wes & $\infty$ \\
\hline $\begin{array}{l}\text { At1g53310 (AtPPC1) } \\
\text { Gene ID: } 841765\end{array}$ & & 5 & $5=$ & 20 & $B=$ & $-\infty$ & 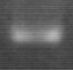 & 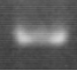 \\
\hline $\begin{array}{l}\text { At3g52930 (FBA, putative) } \\
\text { Gene ID: } 824459\end{array}$ & $-\infty$ & $6=$ & -5 & $=$ & $=$ & 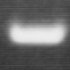 & 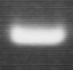 & 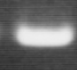 \\
\hline $\begin{array}{l}\text { At5g16050 (GRF5) } \\
\text { Gene ID: } 831462\end{array}$ & 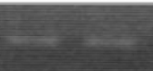 & - & S & - & $\omega$ & $\infty$ & - & 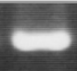 \\
\hline $\begin{array}{l}\text { At1g68750 (AtPPC4) } \\
\text { Gene ID: } 843206\end{array}$ & & & & thes & & & $\infty$ & \\
\hline $\begin{array}{l}\text { At1g64740 (TUA1) } \\
\text { Gene ID: } 842782\end{array}$ & & & & 8 & $5=$ & -4 & 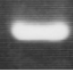 & $=$ \\
\hline $\begin{array}{l}\text { At3g02230 (RGP1) } \\
\text { Gene ID: } 821233\end{array}$ & & & & Es & & co & $\Leftrightarrow$ & - \\
\hline $\begin{array}{l}\text { At2g38210 (PDX1L4) } \\
\text { Gene ID: } 818400\end{array}$ & Fis & 0.5 & 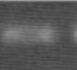 & $\Leftrightarrow$ & & nat & B & se \\
\hline $\begin{array}{l}\text { At3g02090 (MPPBETA) } \\
\text { Gene ID: } 821084\end{array}$ & E & -2 & $\sin$ & $\operatorname{sen}$ & cos & 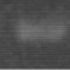 & 5 & - \\
\hline $\begin{array}{l}\text { At3g19950 (zinc finger family protein) } \\
\text { Gene ID: } 821533\end{array}$ & 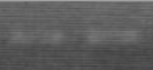 & t. & 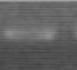 & $\infty$ & $-\infty$ & - & - & 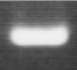 \\
\hline $\begin{array}{l}\text { At3g20250 (APUM5) } \\
\text { Gene ID: } 821569\end{array}$ & & & & & & $5=$ & $-\infty$ & 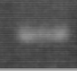 \\
\hline $\begin{array}{l}\text { At1g11410 (T23J18.8) } \\
\text { Gene ID: 837683 }\end{array}$ & & & & & & Se & & $-\infty$ \\
\hline $\begin{array}{l}\text { At1g11340 (T28P6.1) } \\
\text { Gene ID: } 837676\end{array}$ & E & & $E=$ & 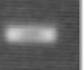 & $c=$ & - & G & 0 \\
\hline $\begin{array}{l}\text { At4g05050 (UBQ11) } \\
\text { Gene ID: } 825847\end{array}$ & & -0 & - & - & Las & Cos & a & - \\
\hline
\end{tabular}

Figure 1 Expression pattern of the putative orthologous genes in various Arabidopsis organs determined by RT-PCR analysis. AGl Code, gene name, and gene ID for each Arabidopsis orthologue are represented by bold letters, shown in parentheses, and found on the 2 nd line, respectively. Ubiquitin 11 (UBQ11, At4g05050) was used as the standard. 1, seedlings; 2, roots; 3, rosette leaves; 4, lateral leaves; 5, stems; 6, flowers; 7, sepals; 8 , petals; 9 , stamens; 10, pistils. 
the small available database of lily proteins. Nineteen distinct orthologous Arabidopsis genes were determined based on the database (Additional file 4). Five candidate proteins (vacuolar $\mathrm{H}^{+}$-ATPase subunit, F1 ATPase, fructose-bisphosphate aldolase-like protein, pyridoxine biosynthesis protein-like, and metalloendopeptidase) have been reported in the Arabidopsis pollen proteomes [14,15]. Moreover, two orthologous Arabidopsis genes (TAIR: At1g78900 and At3g02230) have been reported to be essential for pollen development $[16,17]$.

To determine the expression patterns of the identified candidate Ub-related proteins, the mRNA expression of putative Arabidopsis orthologues were examined because of the limited genomic information available for the lily. Fourteen orthologous genes were selected for RT-PCR analysis in various vegetative tissues, and all of the genes investigated were expressed in flowers. The expression of each gene was examined in the flower organs. All the genes were expressed in the stamen (Figure 1). Among them, the transcripts of $\alpha$-tubulin 1 (TUA1; TAIR At1g64740) and AtBTPC (Atppc4; TAIR At1g68750) showed almost stamen-specific expression. TUA1 is already known to be expressed specifically in pollen $[18,19]$. Therefore, we focused our attention on BTPC. We found that one of the PTPC orthologous genes, Atppc1 (TAIR: At1g53310), is also expressed in stamens (Figure 1). PTPC has also been identified as a $\mathrm{Ub}$-related protein and they are reported to interact in COS $[7,8]$, so both types of PEPC were investigated in the following analysis.

To verify that BTPC and PTPC are Ub-related proteins, lily anther proteins were immunoprecipitated with anti-Ub antibody (FK2; Nippon Bio-Test Laboratories, Tokyo, Japan), which selectively recognizes the Ub moiety but not free Ub [20]. As expected, both bands representing LIBTPC and LIPTPC, of the expected sizes (see Figure $2 \mathrm{~A}$ and $2 \mathrm{~B}$ for the specificities of the antibodies), were co-immunoprecipitated with $\mathrm{FK} 2$, indicating that they are the Ub-related proteins in the lily anther (Figure $2 \mathrm{C}$ ). Another band, larger than the expected size of LIPTPC, was also detected with the anti-AtPTPC antibody (asterisks in Figure 2B and $2 \mathrm{C}$ ). The 'larger' antiAtPTPC antibody immunoreactive band was approximately $8 \mathrm{kDa}$ larger than the 'smaller' immunoreactive band and showed similar mobility to the monoubiquitinated PTPC (Ub-PTPC) band in germinated COS [11]. The larger bands were also co-immunoprecipitated with FK2, but not with mouse serum (Figure 2C), indicating that the larger bands were probably Ub-LIPTPCs.

Large-scale purification enabled the identification of truncated sizes of BTPC and PTPC (Additional files 3 and 4), indicating that the in vitro proteolytic cleavage of the two proteins occurred during protein extraction and purification (these procedures require approximately

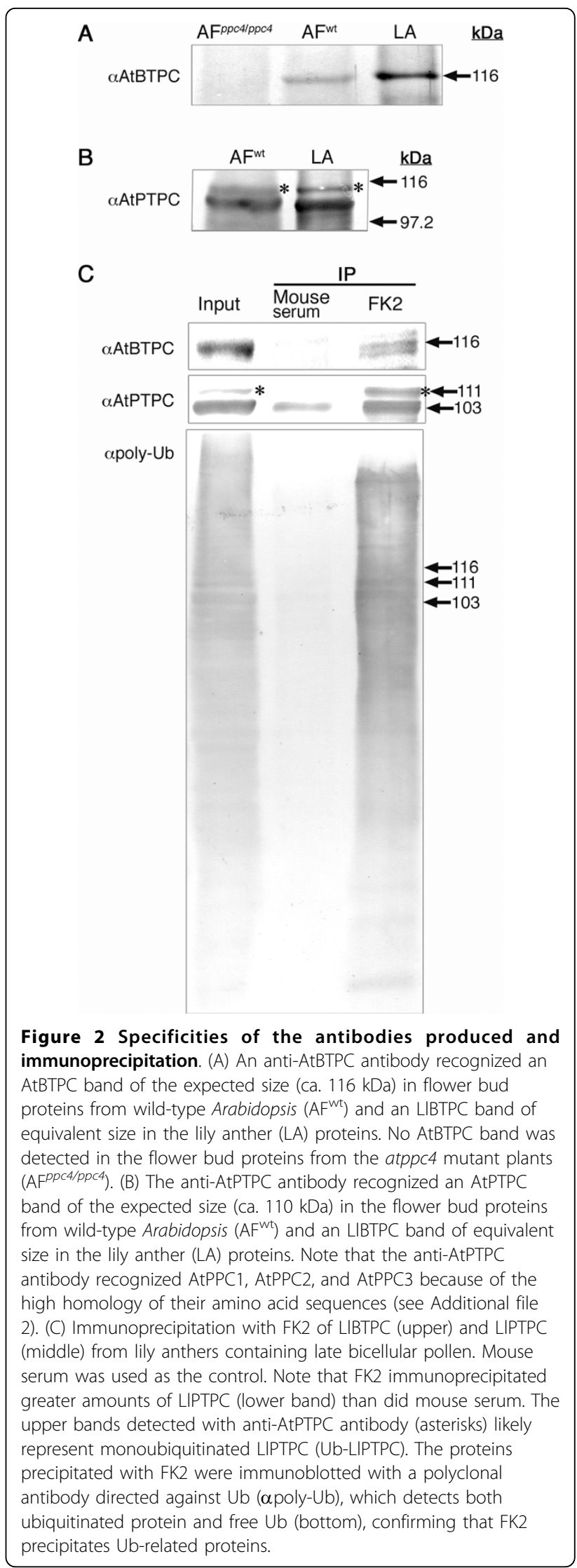


10 hours). In contrast, immunoprecipitated Ub-related proteins contained the expected sizes of BTPC and PTPC (Figure 2C). The size discrepancies of purified BTPC and PTPC between these two immuno-purification experiments may be due to the different purification periods because in vitro COS BTPC proteolysis in an inappropriate buffer was also observed when the incubation period was extended [7]. It seemed like that the anti-AtBTPC antibody detected two bands (Figure 2C), implying the truncation of BTPC by proteolysis or post-translational modification (e.g., monoubiquitination or phosphorylation), although these possibilities need to be confirmed in future studies.

\section{LIBTPC is specifically expressed in pollen after pollen mitosis I (PMI) and forms a complex with LIPTPC} To clarify the tissue specificity of LIBTPC and LIPTPC within the lily anther, the anthers of $14 \mathrm{~cm}$ flower buds were dissected into the anther wall and pollen, and an immunoblot analysis was performed with anti-AtBTPC and anti-AtPTPC antibodies. As shown in Figure 3A, LIBTPC specifically existed in the pollen, whereas LIPTPC was detected in both pollen and the anther wall.

The expression of both PEPCs was examined in pollen at various developmental stages. During pollen development, an asymmetric division of the haploid microspores, called PMI, produces two differently fated cells, a larger VC and a smaller generative cell (GC). The GC, which is enclosed within the $\mathrm{VC}$, undergoes a second mitotic division called "pollen mitosis II" (PMII), giving rise to two sperm cells (SCs) before fertilization [21,22]. PMII in the lily pollen occurs after pollen germination, so the mature pollen is bicellular at anthesis. The lily pollen developmental stages are distinguishable based on the flower bud length [23] (Figure 3B, top diagram). Flower buds of 3-5 cm contain microspores, and PMI is completed by the $7 \mathrm{~cm}$ flower bud stage [24]. The GC morphology changes from round to spindle-like shape in the $12-14 \mathrm{~cm}$ stages [25], and the pollen reaches maturity at anthesis. Immunoblot analysis of LlBTPC during pollen development demonstrated that LIBTPC accumulation starts after PMI, and the amount increases to the $12 \mathrm{~cm}$ flower bud stage, and is sustained at this level until after anthesis (Figure 3B). LIPTPC and UbLIPTPC remained at constant levels during pollen development.

To investigate the interaction between the LIBTPC and LIPTPC proteins and their PEPC activity in pollen, the lily pollen proteins were separated by nondenaturing polyacrylamide gel electrophoresis (PAGE), followed by in-gel staining for PEPC activity and immunoblot analysis (Figure $3 \mathrm{C}$ ). In the microspores at the $3 \mathrm{~cm}$ flower bud stage, the band showing PEPC activity only reacted with the anti-AtPTPC antibody. In pollen from the
$14 \mathrm{~cm}$ flower buds, two PEPC active bands were detected and the smaller band only reacted with the anti-AtPTPC antibody, whereas the larger band reacted with both the anti-AtPTPC and anti-AtBTPC antibodies. The molecular masses of the smaller and larger bands were approximately 450 and $900 \mathrm{kDa}$, respectively. This indicates that LIBTPC interacts with LIPTPC, forming an active PEPC complex in late bicellular pollen.

\section{AtBTPC promoter is preferentially active in pollen}

Because the lily is refractory to molecular analysis, the expression patterns of Arabidopsis PEPC orthologues were analyzed of detail in plant tissues. The Arabidopsis PEPC gene family contains three PTPC genes (Atppc1 [TAIR: At1g53310], Atppc2 [TAIR: At2g42600], and Atppc3 [TAIR: At3g14940]) and one BTPC gene (Atppc4 [TAIR: At1g68750]; Additional file 2). Transgenic plants were produced in which the $\beta$-glucuronidase (GUS) reporter gene was placed under the control of the promoter of each Atppc gene. To roughly compare the expression levels of these genes, all the transgenic lines were incubated with the substrate for the same time. As expected, the AtBTPC gene promoter ProAtppc4 showed significant activity in mature pollen (Figure 4P). Strong GUS staining was detected in the stamens in the late flower bud stage, whereas it was never observed in the younger flower buds (Figure 4O). Faint activity was observed in the root cortices of the same transgenic lines (Figure $4 \mathrm{~N}$ ), but no signal was observed in the leaves (Figure 4M), even with longer incubation. Transgenic plants expressing green fluorescent protein (GFP) under the control of ProAtppc4 showed specific GFP signal in the pollen within the stamens (data not shown), indicating that AtBTPC is expressed specifically in pollen. Conversely, weaker GUS staining was observed in the mature pollen in all transgenic lines when the GUS reporter gene was driven by the AtPTPC gene promoters (ProAtppc1-3). Among the AtPTPCs, ProAtppc3 activity seemed to be strongest in the stamen and pollen in the late flower bud stage (Figure 4C-D, G-H, and 4K-L). ProAtppc1 activity was negligible in mature pollen (Figure 4D), whereas GUS staining was observed in the stamens of the younger flower buds (Figure 4C). All the AtPTPC gene promoters were active in all the somatic tissues investigated here, but with different expression patterns (Figure 4AC, E-G, and 4I-K).

\section{AtBTPC localizes in vegetative cell cytoplasm and shows limited expression during pollen development}

To analyze the localization and the expression pattern of AtBTPC in the pollen during pollen development, "fluorescent tagging of full-length proteins technology" was applied, because this strategy was available to 


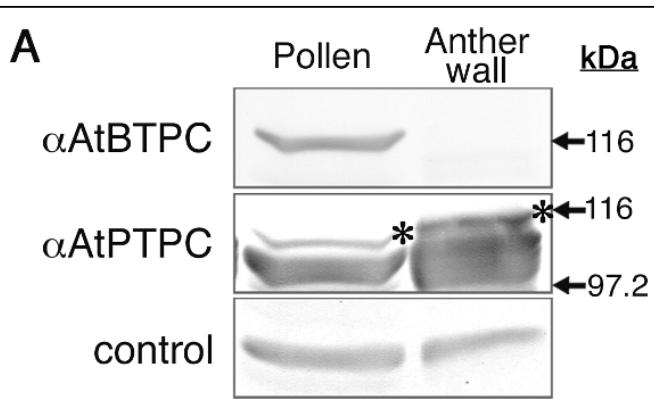

B

Lily pollen development
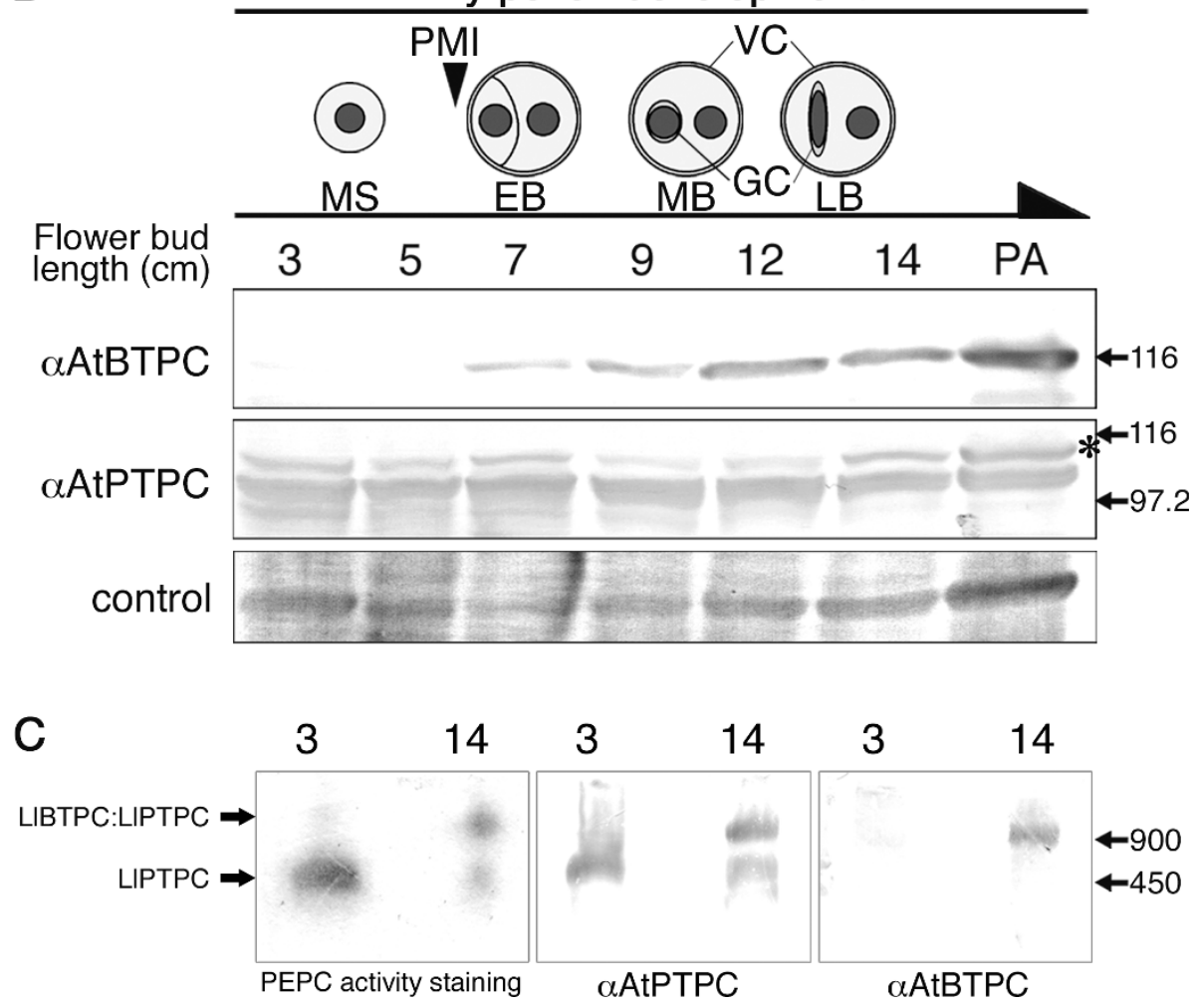

Figure 3 Expression analysis of lily PEPCs in male tissues and developing pollen. (A) Expression of LIBTPC and LIPTPC in anther tissues. Proteins $(10 \mu \mathrm{g})$ from the pollen and anther wall of $14 \mathrm{~cm}$ flower buds were treated with anti-AtBTPC (upper) or anti-AtPTPC (middle) antibody. Non-specific reactive bands were used as loading control (bottom). The upper bands detected with anti-AtPTPC antibody (asterisk) represent UbLIPTPC. (B) Expression patterns of LIBTPC and LIPTPC in developing microspores and pollen. Top diagram illustrates lily pollen development, each stage of which corresponds to the length of the flower bud $(\mathrm{cm})$. Protein extracts from lily pollen $(10 \mu \mathrm{g})$ were subjected to immunoblot analysis with anti-AtBTPC (upper) or anti-AtPTPC (middle) antibody. The upper bands detected with anti-AtPTPC antibody (asterisk) represent UbLIPTPC. Non-specific reactive bands were used as loading control (bottom). PMI, pollen mitosis l; MS, microspore; EB, early bicellular pollen; MB, mid bicellular pollen; LB, late bicellular pollen; GC, generative cell; VC, vegetative cell; PA, post anthesis. (C) Nondenaturing PAGE analysis of microspore and late bicellular pollen proteins $(7 \mu \mathrm{g})$. The numbers in each panel represent the length of the flower bud $(\mathrm{cm})$ containing the microspores (3) or late bicellular pollen (14). In-gel staining for PEPC activity (left), and immunoblot analysis with anti-AtPTPC (middle) or antiAtBTPC (right) antibody were performed.

monitor the expression patterns and subcellular localization of Arabidopsis gene products in planta [26]. The transgenic plants were produced by introducing the native genomic Atppc4 gene, including the 5' and 3' untranslated regions (UTRs), coupled to Venus [27], which encodes a variant of yellow fluorescent protein, with low sensitivity to $\mathrm{pH}$ (Figure 5A). Unlike lily pollen, Arabidopsis pollen undergoes PMII before pollen germination, so the mature pollen is tricellular at anthesis. The stages of pollen development were distinguishable based on the flower bud length (Figure 5B). A notable fluorescent signal was observed in pollen at the $1.5 \mathrm{~mm}$ 

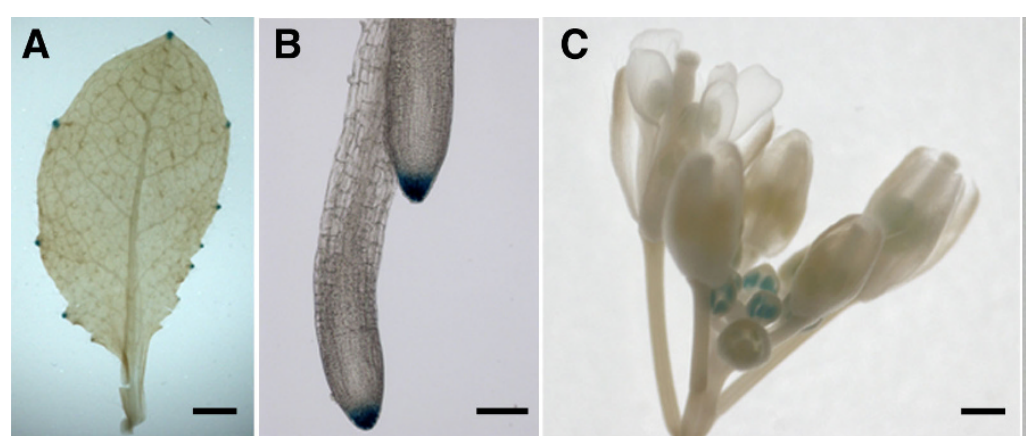

D
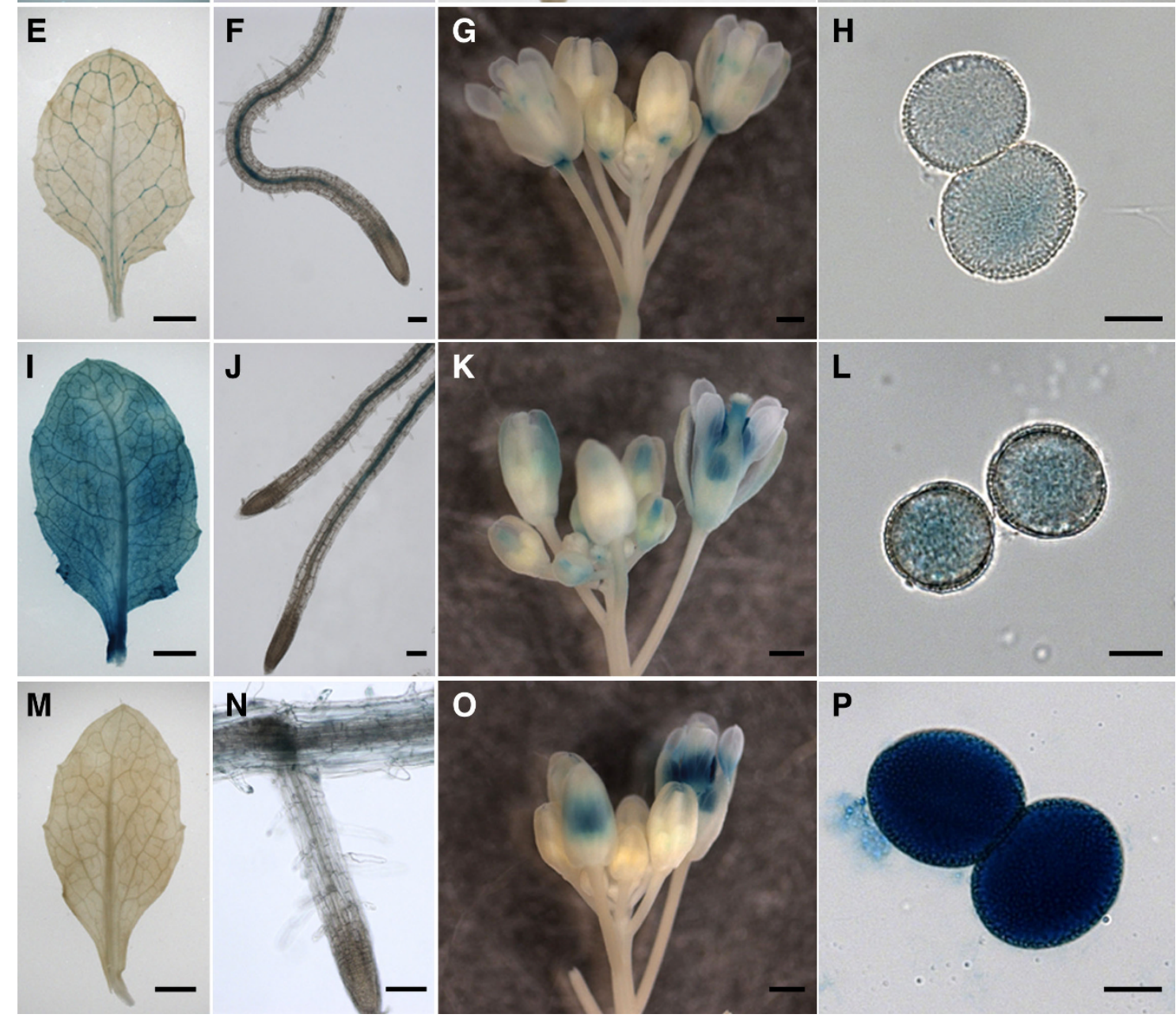

Figure 4 Activities of Atppc1-4 promoters in different organs. Histochemical localization of GUS reporter gene expression in rosette leaf (A, $E, I, M)$, root $(B, F, J, N)$, flower buds $(C, G, K, O)$, and mature pollen $(D, H, L, P)$ driven by the Atppc1 (A-D), Atppc2 (E-H), Atppc3 (I-L), or Atppc4 (M-P) promoter. Bars $=0.5 \mathrm{~mm}$ in $\mathrm{A}, \mathrm{C}, \mathrm{E}, \mathrm{G}, \mathrm{I}, \mathrm{K}, \mathrm{M}$, and $\mathrm{O} ; 50 \mu \mathrm{m}$ in $\mathrm{B}$ and $\mathrm{N} ; 100 \mu \mathrm{m}$ in $\mathrm{F}$ and $\mathrm{J}$; and $10 \mu \mathrm{m}$ in $\mathrm{D}, \mathrm{H}, \mathrm{L}$, and $\mathrm{P}$.

flower bud stage (1.5FB; Figure 5B, E, and 5G). 4', 6-Diamidino-2-phenylindole (DAPI) staining indicated that pollen at $1.5 \mathrm{FB}$ was tricellular (Figure 5D), whereas pollen preceding the $1.2 \mathrm{~mm}$ flower bud stage (1.2FB; Figure 5B) was bicellular (Figure 5C). Confocal laser scanning microscopic (CLSM) analysis of the pollen at 1.5FB showed that AtBTPC-Venus localized in the VC cytoplasm, whereas there was no signal in the $\mathrm{VC}$ nuclei or SCs (Figure 5E).

The levels of AtBTPC-Venus expression during pollen development were estimated by analyzing the fluorescent signal intensities (Figure 5F-I). Whereas no fluorescent signal was observed in the pollen at 1.2FB (Figure 5F), the signal with the most intense fluorescence appeared in 

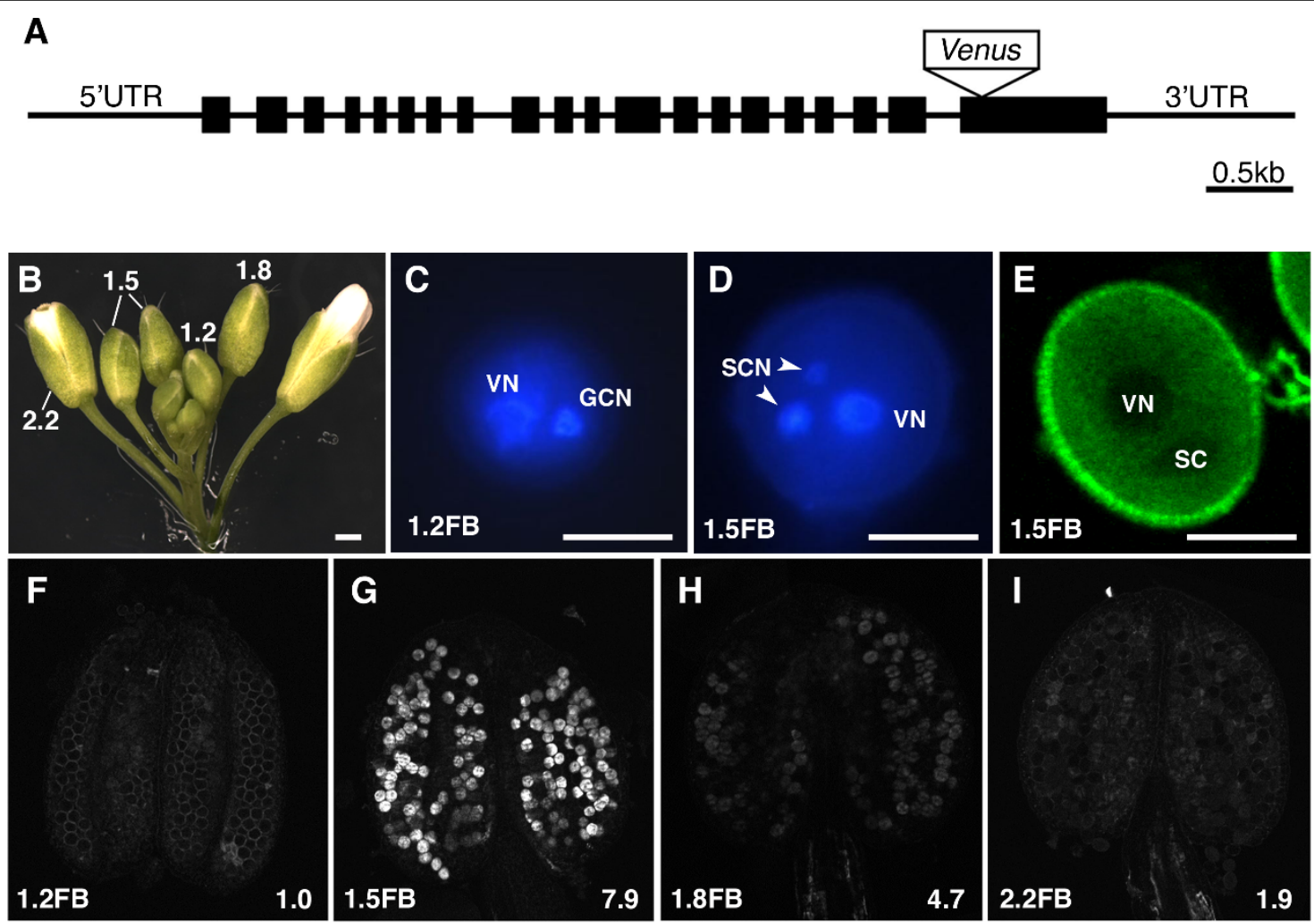

$\mathbf{J}$

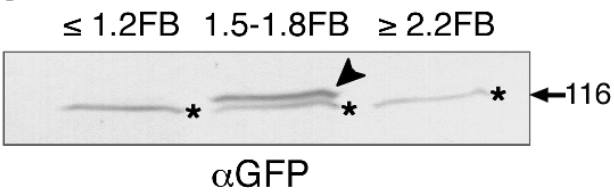

K

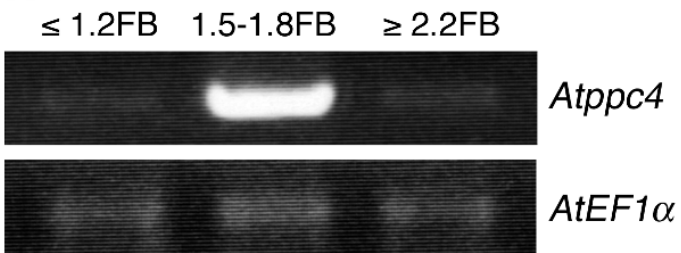

Figure 5 Protein and gene expression of AtBTPC. (A) Schematic view of the gene structure of genomic Atppc4 (At1g68750) used for expression analysis. Black boxes represent exons. The Venus gene was inserted at the Bst1107l site in the last exon. (B) Arabidopsis flower buds. The numbers indicate the flower bud length $(\mathrm{mm})$. Scale bar $=0.5 \mathrm{~mm}$. (C, D) DAPI staining of pollen in $1.2 \mathrm{~mm}$ flower bud $(1.2 \mathrm{FB} ; \mathrm{C})$ and in 1.5 mm flower bud (1.5FB; D). VN, vegetative cell nucleus; GCN, generative cell nucleus; SCN, sperm cell nucleus. Scale bar $=10 \mu \mathrm{m}$. (E) CLSM image of Venus fluorescence in pollen at stage 1.5FB. The blank regions correspond to VN and SC. Scale bar $=10 \mu \mathrm{m}$. (F-I) CLSM images of Venus fluorescence in pollen within an anther at stages 1.2FB-2.2FB. Hemizygous T3 plants were used for the analysis. Each number in the bottom right corner represents the relative fluorescence intensity. Note that the relative fluorescence intensity of the pollen at 1.2FB is given as 1.0, because there was no fluorescent signal in the transgenic pollen at this stage. (J) Immunoblot analysis with anti-GFP antibody of flower buds from homozygous T3 plants. Flower buds were analyzed at stages before 1.2FB $(\leq 1.2 \mathrm{FB})$ at 1.5FB-1.8FB, and after 2.2FB $(\geq 2.2 \mathrm{FB})$. The arrowhead indicates a band of AtPPC4-Venus protein. The lower bands with star marks in all lanes are nonspecific bands. (K) RT-PCR of Atppc4 in flower buds at different stages from wild-type plants. The stages of the flower buds are the same as in (J). Arabidopsis elongation factor $1 \alpha(A t E F 1 \alpha$, TAIR: At1g07920) was used as the standard.

the pollen at 1.5FB (Figure 5G). The intensity then decreased sharply in the later developmental stages (Figure $5 \mathrm{H}$ and $5 \mathrm{I}$ ).

Immunoblot analysis of AtBTPC-Venus using antiGFP antibody showed that the AtBTPC-Venus protein accumulated in the pollen at $1.5-1.8 \mathrm{FB}$, supporting the results of the CLSM analysis (Figure 5J). To confirm the mRNA expression of endogenous AtBTPC in pollen, RT-PCR of full-length Atppc4 was performed. As expected, the Atppc4 transcripts accumulated strongly in the pollen at 1.5-1.8FB, similar to the AtBTPC-Venus protein (Figure $5 \mathrm{~K}$ ).

\section{Discussion}

RT-PCR and promoter-GUS analyses of Arabidopsis PEPC genes revealed that AtBTPC was also preferentially expressed in stamen (Figure 1), particularly in pollen (Figure 4P), similar to lily, whereas AtPTPCs 
(AtPPC1-3) were expressed more strongly in somatic tissues. Unlike a previous study [6], however, the Atppc1 expression shown by our RT-PCR analysis was negligible in somatic tissues (Figure 1). This may be due to the reaction conditions or the developmental stages of the investigated tissues, because transgenic plants with ProAtppc1-GUS showed GUS staining in confined regions of leaf, root, and flower (Figure 4A-C). The difference in the ProAtppc3-GUS expression pattern from the RT-PCR result reported by Sánchez et al. [6] may be due to the same reason as that stated above and/or differences in mRNA and protein stability. Analysis with a reporter protein fused to each Arabidopsis PEPC would provide detailed information of AtPEPC expression.

BTPC is significantly expressed in the pollen of both Arabidopsis and lily (Figures 3A and 4), but the initiation of the expression of each BTPC differs during pollen development (Figures 3B and 5). LIBTPC starts to accumulate after GC formation, after PMI, whereas AtBTPC expression starts immediately after SC formation, after PMII. Based on these findings, it seems that the initiation of BTPC expression in pollen does not depend on the mitosis type, but is triggered after the 'last mitosis' during pollen development, before germination. In both bicellular and tricellular pollen, the expansion of the pollen grain, organelle differentiation, and dehydration occur after the last mitosis for pollen maturation [28]. However, pollen dehydration does not seem to affect Atppc4 expression, because Atppc4 expression started after PMII, but was not sustained until anthesis (Figure 5), although it has been reported that Atppc4 expression is inducible by drought stress in root tissues [29].

We examined the AtBTPC-null mutant line, atppc4 (Figure 2A), but no abnormal characteristics were observed in the mutant pollen when analyzed by DAPI staining and ultrastructural observation, or in plant growth or fertility (data not shown). Therefore, plant BTPC may not be an essential factor for pollen development or plant survival, at least under our experimental growth conditions. Nevertheless, the expression of BTPC is significant in the pollen, suggesting that BTPC plays an important role in pollen development.

It is well known that PEPC is a PTPC homotetramer. In a previous study with COS, the presence of low- and high-molecular-mass PEPC isoforms was reported, which were designated Class-1 (PTPC homotetramer, $410 \mathrm{kDa}$ ) and Class-2 PEPCs (PTPC:BTPC heterooctamer, $910 \mathrm{kDa})$, respectively [7,30]. After nondenaturing PAGE in our study, the small active PEPC band only reacted with anti-AtPTPC antibody, whereas the large active PEPC band reacted with both anti-AtPTPC and anti-AtBTPC antibodies (Figure 3C), showing the same immunoreactivities as COS Class-1 and Class-2 PEPCs, respectively [7]. The small and large active PEPC bands in lily showed similar sizes to those of the COS Class-1 and Class-2 PEPCs, respectively. Therefore, the lily PEPCs in the microspores and the late bicellular pollen exist as complexes, and the small and large PEPC complexes probably corresponded to the COS Class-1 and Class-2 PEPCs, respectively. It has also been reported that PTPC is monoubiquitinated in COS, resulting in a Class-1 PEPC heterotetramer comprised of Ub-PTPC: PTPC [11]. In this work, FK2 immunoprecipitated UbLIPTPC from the lily anther containing late bicellular pollen in addition to the native forms of LIBTPC and LIPTPC (Figure 2C), suggesting that the native forms of LIBTPC and LIPTPC are the associated proteins of ubiquitinated-LIPTPC resulting in the identification by large-scale purification of Ub-related proteins from lily anther (Additional file 3). In addition, Ub-LIPTPC was present together with LIPTPC in the pollen throughout its development from microspore to mature pollen (Figure 3B). From these results, we infer that the Class1 PEPC complex in the microspore comprises UbLIPTPC:LIPTPC and the Class-2 PEPC complex in the late bicellular pollen comprises LlBTPC:Ub-LIPTPC: LIPTPC.

PEPC is allosterically activated by Glc-6-P and inhibited by L-malate and aspartic acid metabolized from oxaloacetate (Additional file 1). Recently, it was reported that Class-2 PEPCs show much lower sensitivity to allosteric activators and inhibitors than do Class-1 PEPC homotetramers, suggesting that the interaction of PTPC and BTPC stabilize the metabolic flow under physiological conditions that would otherwise inhibit Class-1 PEPC [30-32]. It has also been reported that Class-1 PEPC heterotetramers of Ub-PTPC:PTPC are more sensitive to both activators and inhibitors than are Class-1 PEPC homotetramer of PTPC in COS [11]. These reported features and our results together suggest that expressed BTPC binds to the Ub-PTPC:PTPC complex, and that the resulting BTPC:Ub-PTPC:PTPC complex is even less sensitive to inhibitors, but maintains its higher sensitivity to activators relative to the sensitivity of Class-1 PEPC homotetramers. Consequently, it is speculated that the BTPC:Ub-PTPC:PTPC complex may stabilize and accelerate the metabolic flow required for lipid and protein synthesis in pollen (Figure 6). The increase in PEPC activity in developing COS endosperm was coincident with the onset of the most active phase of storage oil accumulation [33]. It has also been reported that the protein content of transgenic bean plants expressing a Corynebacterium glutamicum PEPC was elevated in their seeds by up to $50 \%$ [34]. In lily pollen, there is a marked increase in lipid bodies in the VC cytoplasm of the mature pollen compared with those in the pollen before 
PMI [35]. In Arabidopsis tricellular pollen, storage vacuoles accumulate after PMII and disappear from the mature pollen just before anthesis and at anthesis [36]. The patterns of the appearance of storage organelles in lily and Arabidopsis pollen almost coincide with the patterns of LIBTPC and AtBTPC expression, respectively, supporting our hypothesis that BTPC plays a role in the acceleration of metabolic flow to facilitate the synthesis of storage substances during late pollen development. Previous studies of Class-2 PEPCs have suggested that green alga and vascular plants have divergent PEPCs that serve to function in adaptation for survival in different environments [30-32,37]. It will be interesting to determine what triggers and suppresses the expression of BTPC during pollen development. Further investigation of Class-1 and Class-2 PEPCs is expected to provide more information of the metabolic pathway underlying the synthesis of storage substances in both bicellular and tricellular pollens during development.

\section{Conclusions}

The large-scale purification of Ub-related proteins from the lily anther should advance research into the reproductive factors related to Ub-mediated protein modifications. In this study, BTPC was first found as a Ub-related protein in pollen.

LIBTPC and AtBTPC show significant expression in pollen and they are first expressed after the last mitosis before pollen germination. The duration of their expression almost coincides with the appearance of storage organelles during the maturation processes of both pollens. The presence of BTPC:Ub-PTPC:PTPC with PEPC activity during pollen maturation is also suggested in this study. It would be necessary to confirm the presence of the Ub-PTPC:PTPC and BTPC:Ub-PTPC:PTPC complexes with their kinetic properties in pollen in future studies. Furthermore, future tasks include clarification of the AtPTPC proteins (AtPPC1-3) involved in Class- 1 and Class-2 PEPC formation during pollen development because AtPTPCs show different expression patterns (Figure 2), and investigation of their posttranslational modifications, such as ubiquitination and phosphorylation. The results are expected to shed light on the role of PEPCs in pollen development.

BTPC is strongly expressed in the late stage of pollen development, but an AtBTPC-deficient mutant plant, atppc4, showed no abnormalities in pollen development, at least under our experimental growth conditions. PEPC itself is involved in the anaplerotic replenishment of intermediates of the TCA cycle, and for this reason, any obvious effects of BTPC deficiency may be difficult to identify. Alternatively, analysis under various physiological and growth conditions, such as varying temperatures, may identify the physiological function of BTPC in the reproductive process, because the thermal stability of Class-2 PEPC activity is relatively increased [32]. This is the first characterization of BTPC in pollen, the male gametophyte of higher plants. Our work provides new information for the study of the function of BTPC in plants.

\section{Methods}

\section{Primers}

Information on all primers used in this study is given in Additional file 5 .

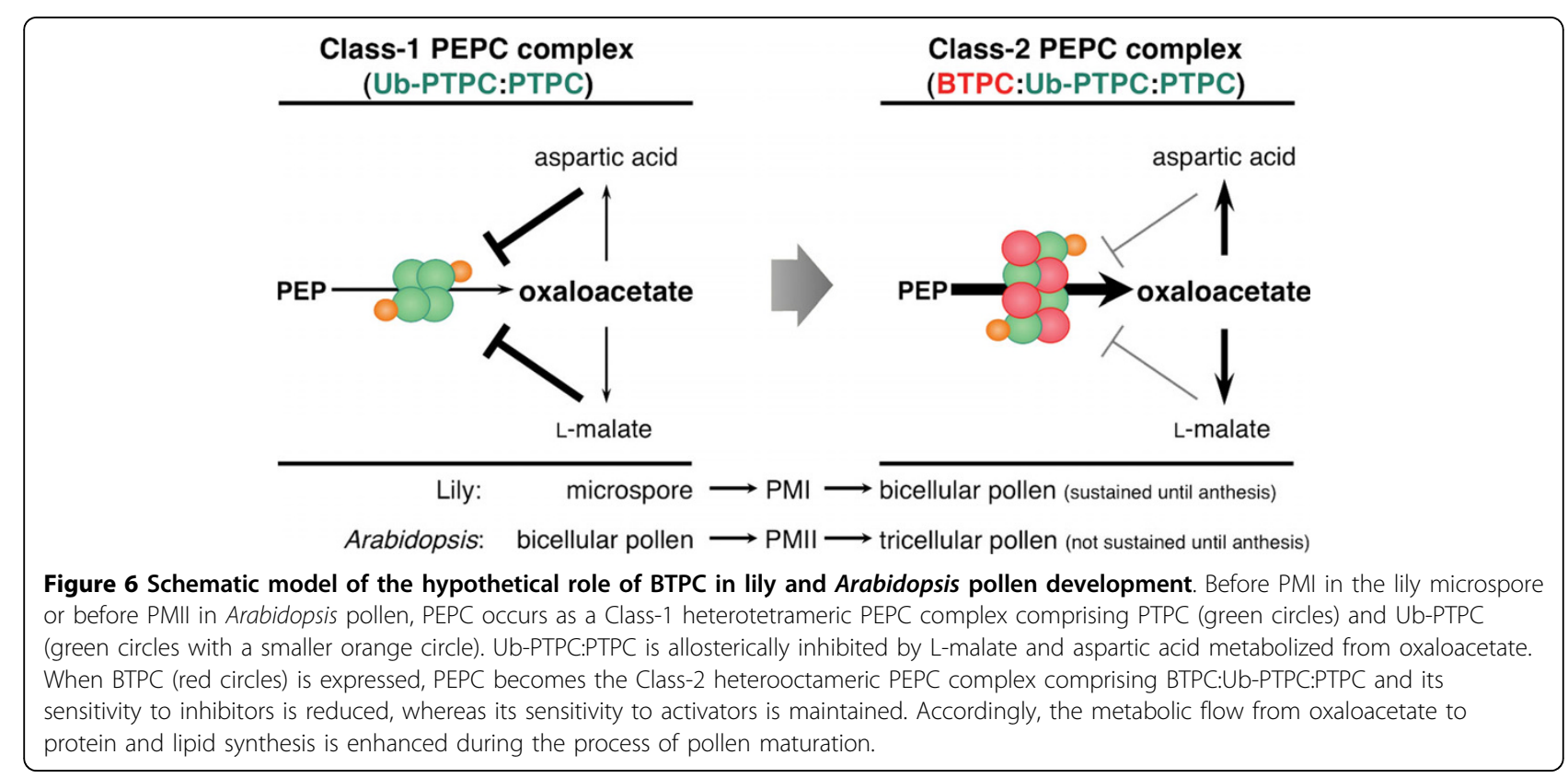




\section{Plant materials}

Trumpet lily (Lilium longiflorum cv. Hinomoto) was grown in a greenhouse for immunoblot analysis and nondenaturing PAGE.

Arabidopsis thaliana (ecotype Columbia 0) was grown at $22^{\circ} \mathrm{C}$ under a $16 / 8 \mathrm{~h} \mathrm{light/dark} \mathrm{cycle.} \mathrm{A} \mathrm{Salk} \mathrm{line}$ (SALK_144112; background Columbia) of Atppc4 was obtained from the Arabidopsis Biological Resource Center http://abrc.osu.edu/. The genotype of this line was confirmed by PCR using the gene-specific primer 'ppc4SALK-For' and the T-DNA-specific primer 'LBc'. The T-DNA insertion site was verified by sequencing.

\section{Large-scale purification and identification of Ub-related proteins in lily anther}

Anthers of 12-14 cm flower buds were collected from commercially obtained trumpet lily (L. longiflorum). Approximately $100 \mathrm{mg}$ of total crude protein was obtained from 150 lily anthers. Protein extraction, purification with an anti-Ub antibody (FK2), in-gel digestion, and LC-MS/MS analysis were performed as described by Igawa et al. [13]. The MS/MS spectra were analyzed with the MASCOT search engine (MatrixScience, http:// www.matrixscience.com) against an NCBI protein database of all plant species. The peptide sets were then summarized manually, and proteins encoding ubiquitin and proteasomal subunits were eliminated from the list.

\section{RT-PCR}

Total RNA was prepared from various Arabidopsis organs using TRIzol ${ }^{\odot}$ Reagent (Life Technologies Japan, Ltd., Tokyo, Japan). cDNA was synthesized from $1 \mu \mathrm{g}$ of total RNA with oligo $(\mathrm{dT})_{20}$ primer using the Rever-Tra Ace- $\alpha$ kit (Toyobo Co., Ltd., Osaka, Japan).

\section{Antibodies, immunoblot analysis, and immunoprecipitation}

To produce polyclonal anti-AtPTPC and anti-AtBTPC antibodies, the open reading frames encoding the 300 $\mathrm{N}$-terminal amino acids of AtPPC1 (At1g53310) and AtPPC4 (At1g68750; Additional file 2) were amplified using the cDNA derived from seedlings and stamens, respectively. Primers 'PPC1-3AB-F' and 'PPC1-3AB-R' for Atppc1 and primers 'PPC4AB-F' and 'PPC4AB-R' for Atppc4 were used to add NdeI sites to the $5^{\prime}$ termini and BamHI sites to the $3^{\prime}$ termini. The NdeI-BamHI fragments of Atppc1 and Atppc4 were cloned into the expression vector pET28c (+) encoding $6 \times$ histidine at the $\mathrm{N}$ terminus. The plasmids generated were transformed into BL21 (DE3) cells. The purified histidinetagged AtPPC1 and AtPPC4 were injected into rabbits as antigens. The purified antisera were designated antiAtPTPC and anti-AtBTPC antibodies, respectively. AntiGFP antibody (Living Colors ${ }^{\circledR}$ A. v. Monoclonal
Antibody [JL-8]; Takara Bio Inc., Otsu, Japan) and polyclonal anti-Ub antibody (Sigma-Aldrich, Missouri, USA) were purchased.

For the immunoblot analysis, lily pollen and Arabidopsis flower buds were ground in liquid $\mathrm{N}_{2}$ and homogenized with the buffer described by Gennidakis et al. [7]. The homogenates were centrifuged at $17,400 \times \mathrm{g}$ at $4^{\circ} \mathrm{C}$ for $15 \mathrm{~min}$, and the supernatants were centrifuged again for $5 \mathrm{~min}$ to isolate the total protein extract. Immediately after the extraction, the protein extracts were treated with SDS sample buffer.

For immunoprecipitation with FK2 [20], the lily anther proteins were homogenized with the extraction buffer used for the large-scale purification of Ub-related proteins [13]. The homogenates were centrifuged at $17,400 \times \mathrm{g}$ at $4^{\circ} \mathrm{C}$ for $15 \mathrm{~min}$. The lipid layer was removed and the supernatants were centrifuged again for $5 \mathrm{~min}$ to isolate the total protein extracts. The protein extracts were mixed with Protein A-Sepharose 6MB (Sigma-Aldrich) coupled to $\mathrm{FK} 2$, and then incubated for $3 \mathrm{~h}$ at $4^{\circ} \mathrm{C}$. Immediately after the incubation, the immunoprecipitated proteins were treated with SDS sample buffer.

The molecular masses $(\mathrm{kDa})$ in Figures 2A-B, 3A, B, 5J and Additional file 3 were marked according to the positions of marker proteins (Protein Marker Broad Range; New England BioLabs Inc., MA, USA). The molecular masses in Figure $2 \mathrm{C}$ and Additional file 4 were estimated by calculation based on the mobilities of marker proteins (Prestained Protein Marker Broad Range; Cell Signaling Technology, Inc., MA, USA).

\section{Nondenaturing PAGE and staining for PEPC activity}

The lily pollen extracts were prepared with the buffer described by Uhrig et al. [8] with modifications: the omission of 2,2'-dipyridyl disulfide and the addition of $20 \mu \mathrm{L} /$ $\mathrm{mL}$ Calbiochem's Plant Protease Inhibitor Cocktail (Merck KGaA, Darmstadt, Germany). Nondenaturing PAGE and staining for PEPC activity were as described by Law and Plaxton [38]. The approximate molecular masses (Figure 3C) were estimated by calculation based on the mobilities of marker proteins (NativeMark Unstained Protein Standard; Life Technologies Japan, Ltd.).

\section{Construction of plasmids and generation of Arabidopsis transgenic plants}

ProAtppc1, ProAtppc2, and ProAtppc3 were amplified by genomic PCR and each PCR product was ligated into the $\mathrm{pENTR}^{\mathrm{im}} / \mathrm{D}$-TOPO vector (Life Technologies Japan, Ltd.), then transferred with the LR reaction (Gateway; Life Technologies Japan, Ltd.) to the destination vector pGWB203 [39] carrying the GUS reporter gene. ProAtppc4 was amplified with primers 'ppc4-promoterF' and 'ppc4-promoterR', which added a SalI site to the $5^{\prime}$ terminus and a BamHI site to the $3^{\prime}$ terminus. The SalI-BamHI fragment 
was cloned into pENTR ${ }^{\mathrm{rm}} 3 \mathrm{C}$ (Life Technologies Japan Ltd.), and then transferred into pGW203 by the LR reaction. The genomic region of Atppc4 from the translation start site to the $3^{\prime}$ UTR (ca. $1 \mathrm{~kb}$ ) was amplified with primers 'PPC4-1F' and 'PPC4-3UTR-R', which added a BamHI site to the $5^{\prime}$ terminus and a NotI site to the $3^{\prime}$ terminus. The BamHI-NotI fragment was cloned into pENTR $^{\mathrm{m} 3 \mathrm{C}}$ carrying ProAtppc4. Venus, amplified by PCR with primers 'VenusF2' and 'VenusR2', was inserted at the unique Bst1107I site in the resulting plasmid. Finally, the ProAtppc4::genomic-Atppc4+venus fragment was transferred to pGWB1 by the LR reaction. All the plasmids generated were transformed into Agrobacterium tumefaciens strain GV3101, which was used to infect wild-type Arabidopsis plants.

\section{GUS assays and DAPI staining}

The GUS substrate solution contained $100 \mathrm{mM} \mathrm{NaPO}_{4}$ (pH 7.0), $10 \mathrm{mM}$ EDTA, $2.5 \mathrm{mM} \mathrm{K}_{3}\left(\mathrm{Fe}[\mathrm{CN}]_{6}\right), 2.5 \mathrm{mM}$ $\mathrm{K}_{4}\left(\mathrm{Fe}[\mathrm{CN}]_{6}\right), 0.1 \%$ Triton X-100, and $0.5 \mathrm{mg} / \mathrm{L} \mathrm{X-Gluc.}$ The samples were incubated in the dark at $37^{\circ} \mathrm{C}$ for $1 \mathrm{~h}$ (pollen) or $5 \mathrm{~h}$ (leaf, root, and inflorescence). The pollen was stained with DAPI as described by Park et al. [40].

\section{Microscopy and image analysis}

Specimens of GUS-assayed Arabidopsis inflorescences and DAPI-stained pollen were observed under a Stemi 2000-C (Carl Zeiss Co., Ltd., Tokyo, Japan) or Axioplan 2 microscope (Carl Zeiss Co., Ltd.), and photo images were captured with an AxioCam MRc using AxioVision software (Carl Zeiss Co., Ltd.).

Anthers at various flower bud stages, taken from hemizygous T3 plants expressing Atppc4-Venus, were observed with the CLSM FV1000 Inverted Confocal Microscope (excitation $458 \mathrm{~nm}$ and emission band path 530-630 nm; Olympus Corp., Tokyo, Japan) with FluoroView software (Olympus Corp.). The fluorescence intensities were quantified with the ImageJ software http://rsbweb.nih.gov/ij/. The average values for the wild-type and transgenic pollens were obtained from at least 20 pollen grains per anther at each developmental stage. The average values for transgenic pollen were then divided by the average value for the wild-type pollen to calculate the relative fluorescence intensities (pollen at 1.2FB showed no fluorescent signal, so the value was given as 1.0).

\section{Additional material}

Additional file 1: Simplified metabolic pathway diagram. This file shows a simplified metabolic pathway diagram for $\operatorname{COS}$ germinating seeds [33]. PEPC catalyzes the irreversible reaction that produces oxaloacetate from PEP. PEPC activation leads to protein and lipid synthesis. PEPC activity is allosterically activated by Glc-6-P and inhibited by L-malate and aspartic acid.
Additional file 2: Amino acid alignment of the AtPEPC family. The amino acid alignment of the AtPEPC family. Residues conserved among four or three paralogues are highlighted with black or gray, respectively. The serine residue marked with the red inverted triangle is a conserved phosphorylation site in PTPC. The red bars above and the blue bars below the alignment indicate the corresponding peptide sequences of AtPTPC and AtBTPC, respectively, which were detected in the lily anther with LC-MS/MS analysis. The amino acid sequences overlain with pale red and pale blue were used to produce the anti-AtPTPC and antiAtBTPC antibodies, respectively.

Additional file 3: Immunopurified proteins with FK2 from lily anther. Proteins immunoprecipitated with FK2 from lily anther were subjected to SDS-PAGE and stained with Flamingo ${ }^{\mathrm{TM}}$ (Bio-Rad Laboratories, CA, USA). Clear bands (marked with lowercase letter) were excised and numbered smearing regions cut into 2-mm-long gel pieces were digested with trypsin for LC-MS/MS analysis.

Additional file 4: Table of Ub-related proteins identified from lily anther and the putative Arabidopsis orthologous genes. This additional file contains a table of the Ub-related proteins identified in the lily anther and the putative Arabidopsis orthologous proteins. Candidate proteins with high reliability (MASCOT score $>40 ; P<0.05$ ) are listed. The gel position for each identified polypeptide corresponds to that in Additional file 3. The approximate size of each identified protein was estimated by calculation based on the mobilities of marker proteins (indicated on the left of the panel in Additional file 3). Each Arabidopsis orthologous protein was determined with a BLASTP search at the TAIR website http://www.arabidopsis.org/Blast/index.jsp based on the protein sequence indicated in the corresponding column. The expression of the genes marked with an asterisk was checked by RT-PCR (see Figure 1).

Additional file 5: Primers used in this study. Primers used in this study.

\section{Acknowledgements}

We are grateful to Dr T. Nakagawa (Shimane University, Japan) for the pGWB1 and pGWB203 vectors, and to Dr A. Miyawaki (RIKEN, BSI, Japan) for the Venus gene. We thank Dr N. Inada (NAIST, Japan) for assistance with the microscopy and for helpful discussions, and Ms R. Okanami for technical assistance. This work was supported by KAKENHI (19658041), a Grant-in-Aid for Exploratory Research from Japan Society for the Promotion of Science (JSPS), KAKENHI (21025001), a Grant-in-Aid for Scientific Research on Priority Areas from Ministry of Education, Culture, Sports, Science and Technology, Japan, and a Grant-in-Aid for Scientific Research from Nara Institute of Science and Technology supported by Ministry of Education, Culture, Sports, Science and Technology, Japan.

\section{Author details}

${ }^{1}$ The Plant Science Education Unit, The Graduate School of Biological Sciences, Nara Institute of Science and Technology, 8916-5 Takayama-cho, Ikoma, Nara 630-0101, Japan. ${ }^{2}$ Graduate School of Nanobioscience, Yokohama City University, 22-2 Seto, Kanazawa-ku, Yokohama 236-0027, Japan. ${ }^{3}$ National Institute of Crop Science, National Agriculture and Food Research Organization, 2-1-18 Kannondai, Tsukuba, Ibaraki 305-8518, Japan. ${ }^{4}$ Current Address: Initiative Research Program, Advanced Science Institute, RIKEN, 2-1 Hirosawa, Wako, Saitama 351-0198, Japan.

\section{Authors' contributions}

$\mathrm{TI}$ and $\mathrm{YY}$ conceived the study and designed all the experiments. IT grew and prepared the lily samples. MF and YF performed the LC-MS/MS analysis. TI performed all other analyses and interpreted the experimental data. TI, IT, and YY participated in writing the manuscript. All the authors have read and approved the final manuscript.

Received: 22 April 2010 Accepted: 14 September 2010

Published: 14 September 2010 


\section{References}

1. Izui K, Matsumura H, Furumoto $T$, Kai $Y$ : Phosphoenolpyruvate carboxylase: a new era of structural biology. Annu Rev Plant Biol 2004, 55:69-84.

2. Chollet $R$, Vidal J, O'Leary MH: PHOSPHOENOLPYRUVATE CARBOXYLASE: A Ubiquitous, Highly Regulated Enzyme in Plants. Annu Rev Plant Physiol Plant Mol Biol 1996, 47:273-298.

3. Nimmo HG: Control of the phosphorylation of phosphoenolpyruvate carboxylase in higher plants. Arch Biochem Biophys 2003, 414:189-196.

4. Podestá FE, Moorhead GB, Plaxton WC: Potato tuber pyrophosphatedependent phosphofructokinase: effect of thiols and polyalcohols on its intrinsic fluorescence, oligomeric structure, and activity in dilute solutions. Arch Biochem Biophys 1994, 313:50-57

5. Podestá FE, Plaxton WC: Regulation of cytosolic carbon metabolism in germinating Ricinus communis cotyledons II. Properties of phophoenolpyruvate carboxylase and cytosolic pyruvate kinase associated with the regulation of glycolysis and nitrogen assimilation. Planta 1994, 194:381-387.

6. Sánchez R, Cejudo FJ: Identification and expression analysis of a gene encoding a bacterial-type phosphoenolpyruvate carboxylase from Arabidopsis and rice. Plant Physiol 2003, 132:949-957.

7. Gennidakis S, Rao S, Greenham K, Uhrig RG, O'Leary B, Snedden WA, Lu C, Plaxton WC: Bacterial- and plant-type phosphoenolpyruvate carboxylase polypeptides interact in the hetero-oligomeric Class-2 PEPC complex of developing castor oil seeds. Plant J 2007, 52:839-849.

8. Uhrig RG, O'Leary B, Spang HE, MacDonald JA, She YM, Plaxton WC: Coimmunopurification of phosphorylated bacterial- and plant-type phosphoenolpyruvate carboxylases with the plastidial pyruvate dehydrogenase complex from developing castor oil seeds. Plant Physiol 2008, 146:1346-1357.

9. Maor R, Jones A, Nuhse TS, Studholme DJ, Peck SC, Shirasu K: Multidimensional protein identification technology (MudPIT) analysis of ubiquitinated proteins in plants. Mol Cell Proteomics 2007, 6:601-610.

10. Saracco SA, Hansson M, Scalf M, Walker JM, Smith LM, Vierstra RD: Tandem affinity purification and mass spectrometric analysis of ubiquitylated proteins in Arabidopsis. Plant J 2009, 59:344-358.

11. Uhrig RG, She YM, Leach CA, Plaxton WC: Regulatory monoubiquitination of phosphoenolpyruvate carboxylase in germinating castor oil seeds. $J$ Biol Chem 2008, 283:29650-29657.

12. Haglund K, Dikic I: Ubiquitylation and cell signaling. EMBO J 2005, 24:3353-3359.

13. Igawa T, Fujiwara M, Takahashi H, Sawasaki T, Endo Y, Seki M, Shinozaki K, Fukao $Y$, Yanagawa $Y$ : Isolation and identification of ubiquitin-related proteins from Arabidopsis seedlings. J Exp Bot 2009, 60:3067-3073.

14. Holmes-Davis R, Tanaka CK, Vensel WH, Hurkman WJ, McCormick S: Proteome mapping of mature pollen of Arabidopsis thaliana. Proteomics 2005, 5:4864-4884.

15. Noir S, Brautigam A, Colby T, Schmidt J, Panstruga R: A reference map of the Arabidopsis thaliana mature pollen proteome. Biochem Biophys Res Commun 2005, 337:1257-1266.

16. Jakobsen MK, Poulsen LR, Schulz A, Fleurat-Lessard P, Moller A, Husted S, Schiott M, Amtmann A, Palmgren MG: Pollen development and fertilization in Arabidopsis is dependent on the MALE GAMETOGENESIS IMPAIRED ANTHERS gene encoding a type V P-type ATPase. Genes DeV 2005, 19:2757-2769.

17. Drakakaki G, Zabotina O, Delgado I, Robert S, Keegstra K, Raikhel N: Arabidopsis reversibly glycosylated polypeptides 1 and 2 are essential for pollen development. Plant Physiol 2006, 142:1480-1492.

18. Carpenter JL, Ploense SE, Snustad DP, Silflow CD: Preferential expression of an alpha-tubulin gene of Arabidopsis in pollen. Plant Cell 1992, 4:557-571.

19. Kim Y, An G: Pollen-specific expression of the Arabidopsis thaliana alpha 1-tubulin promoter assayed by beta-glucuronidase, chloramphenicol acetyltransferase and diphtheria toxin reporter genes. Transgenic Res 1992, 1:188-194.

20. Fujimuro M, Sawada H, Yokosawa H: Production and characterization of monoclonal antibodies specific to multi-ubiquitin chains of polyubiquitinated proteins. FEBS Lett 1994, 349:173-180.

21. McCormick S: Male Gametophyte Development. Plant Cell 1993, 5:1265-1275.

22. McCormick S: Control of male gametophyte development. Plant Cell 2004, 16(Suppl):S142-153.
23. Mori $T$, Kuroiwa $H$, Higashiyama $T$, Kuroiwa $T$ : Identification of higher plant GlsA, a putative morphogenesis factor of gametic cells. Biochem Biophys Res Commun 2003, 306:564-569.

24. Sano $Y$, Tanaka I: A histone H3.3-like gene specifically expressed in the vegetative cell of developing lily pollen. Plant Cell Physiol 2005, 46:1299-1308.

25. Ueda K, Kinoshita Y, Xu ZJ, Ide N, Ono M, Akahori Y, Tanaka I, Inoue M: Unusual core histones specifically expressed in male gametic cells of Lilium longiflorum. Chromosoma 2000, 108:491-500.

26. Tian GW, Mohanty A, Chary SN, Li S, Paap B, Drakakaki G, Kopec CD, Li J, Ehrhardt D, Jackson D, et al: High-throughput fluorescent tagging of fulllength Arabidopsis gene products in planta. Plant Physiol 2004, 135:25-38.

27. Nagai T, Ibata K, Park ES, Kubota M, Mikoshiba K, Miyawaki A: A variant of yellow fluorescent protein with fast and efficient maturation for cellbiological applications. Nat Biotechnol 2002, 20:87-90.

28. Bedinger P: The remarkable biology of pollen. Plant Cell 1992, 4:879-887.

29. Sánchez R, Flores A, Cejudo FJ: Arabidopsis phosphoenolpyruvate carboxylase genes encode immunologically unrelated polypeptides and are differentially expressed in response to drought and salt stress. Planta 2006, 223:901-909.

30. Blonde JD, Plaxton WC: Structural and kinetic properties of high and low molecular mass phosphoenolpyruvate carboxylase isoforms from the endosperm of developing castor oilseeds. J Biol Chem 2003, 278:11867-11873.

31. Rivoal J, Trzos S, Gage DA, Plaxton WC, Turpin DH: Two unrelated phosphoenolpyruvate carboxylase polypeptides physically interact in the high molecular mass isoforms of this enzyme in the unicellular green alga Selenastrum minutum. J Biol Chem 2001, 276:12588-12597.

32. O'Leary B, Rao SK, Kim J, Plaxton WC: Bacterial-type phosphoenolpyruvate carboxylase (PEPC) functions as a catalytic and regulatory subunit of the novel class-2 PEPC complex of vascular plants. J Biol Chem 2009, 284:24797-24805.

33. Sangwan RS, Singh N, Plaxton WC: Phosphoenolpyruvate Carboxylase Activity and Concentration in the Endosperm of Developing and Germinating Castor Oil Seeds. Plant Physiol 1992, 99:445-449.

34. Rolletschek H, Borisjuk L, Radchuk R, Miranda M, Heim U, Wobus U, Weber H: Seed-specific expression of a bacterial phosphoenolpyruvate carboxylase in Vicia narbonensis increases protein content and improves carbon economy. Plant Biotechnol J 2004, 2:211-219.

35. Nakamura $\mathrm{S}$, Miki-Hirosige $\mathrm{H}$ : Fine-structural study on the formation of the generative cell wall and intine-3 layer in a growing pollen grain of Lilium Longiflorum. Amer J Bot 1985, 72:365-375.

36. Yamamoto Y, Nishimura M, Hara-Nishimura I, Noguchi T: Behavior of vacuoles during microspore and pollen development in Arabidopsis thaliana. Plant Cell Physiol 2003, 44:1192-1201.

37. Moellering ER, Ouyang Y, Mamedov TG, Chollet R: The two divergent PEPcarboxylase catalytic subunits in the green microalga Chlamydomonas reinhardtii respond reversibly to inorganic- $\mathrm{N}$ supply and co-exist in the high-molecular-mass, hetero-oligomeric Class-2 PEPC complex. FEBS Lett 2007, 581:4871-4876.

38. Law RD, Plaxton WC: Purification and Characterization of a Novel Phosphoenolpyruvate Carboxylase from Banana Fruit. Biochemical Journal 1995, 307:807-816

39. Nakagawa T, Kurose T, Hino T, Tanaka K, Kawamukai M, Niwa Y, Toyooka $K$, Matsuoka K, Jinbo T, Kimura T: Development of series of gateway binary vectors, pGWBs, for realizing efficient construction of fusion genes for plant transformation. J Biosci Bioeng 2007, 104:34-41.

40. Park SK, Howden R, Twell D: The Arabidopsis thaliana gametophytic mutation gemini pollen 1 disrupts microspore polarity, division asymmetry and pollen cell fate. Development 1998, 125:3789-3799.

doi:10.1186/1471-2229-10-200

Cite this article as: Igawa et al:: Characterization of bacterial-type phosphoenolpyruvate carboxylase expressed in male gametophyte of higher plants. BMC Plant Biology 2010 10:200. 